\title{
Curvature Tensor of the Stationary Accelerated Frame in Gravity Field
}

\author{
Sangwha-Yi*
}

Department of Mathematics, Taejon University, Taejon, South Korea

We define an accelerated frame that moves along $\hat{r}$-axis in the general relativistic curved space-time. We then calculate the curvature tensor of this accelerated frame in the stationary gravity field. The curvature tensor is divided into two parts: the curvature tensor as observed by the observer and the curvature tensor of the observer's own planet in the gravity field.

\section{Introduction}

The objective at first is to define an accelerated frame that moves along $\hat{r}$-axis in the curved space-time. In this context, the Schwarzschild solution is given as

$$
\begin{aligned}
d \tau^{2}=\left(1-\frac{2 G M}{r c^{2}}\right) d t^{2} \\
\\
\quad-\frac{1}{c^{2}}\left[\frac{d r^{2}}{1-\frac{2 G M}{r c^{2}}}+r^{2} d \theta^{2}+r^{2} \sin ^{2} \theta d \phi^{2}\right]
\end{aligned}
$$

Now, the acceleration of moving matter is $a$ in the Schwarzschild space-time, which is given as

$$
a=\frac{d}{d t}\left(\frac{u}{\sqrt{1-\frac{2 G M}{r c^{2}}-\frac{u^{2}}{c^{2}}}}\right)=a_{\text {inertial }}-g
$$

Here, $a_{\text {inertial }}$ is the inertial acceleration, $g$ is the pure gravity acceleration and $\mathrm{u}$ is given as

$$
u=\frac{1}{\sqrt{1-\frac{2 G M}{r c^{2}}}} \frac{d r}{d t}
$$

$$
\text { If } a_{0}=a / \sqrt{1-\frac{2 G M}{r c^{2}}} \text { then }
$$

\footnotetext{
*sangwah1@ @ate.com
}

$$
\begin{aligned}
& a_{0}=\frac{1}{\sqrt{1-\frac{2 G M}{r c^{2}}}} \frac{d}{d t}\left(\frac{u}{\sqrt{1-\frac{2 G M}{r c^{2}}-\frac{u^{2}}{c^{2}}}}\right)=\frac{d}{d \hat{t}}\left(\frac{V}{\sqrt{1-\frac{V^{2}}{c^{2}}}}\right) \\
& V=\frac{d \hat{r}}{d \hat{t}}=\frac{d r}{d t} \frac{1}{\left(1-\frac{2 G M}{r c^{2}}\right)}, \quad d \hat{t}=d t \sqrt{1-\frac{2 G M}{r c^{2}}} \\
& a_{0} \hat{t}=\frac{d \hat{r}=\frac{d r}{\sqrt{1-\frac{2 G M}{r c^{2}}}}}{\sqrt{1-\frac{V^{2}}{c^{2}}}}, \quad V=\frac{a_{0} \hat{t}}{\sqrt{1+\frac{a_{0}{ }^{2} \hat{t}^{2}}{c^{2}}}}
\end{aligned}
$$

Here, $V$ is the velocity of $\hat{r}$-axis velocity

$$
\text { If, } \frac{d \theta}{d t}=\frac{d \phi}{d t}=0 \text {, the solution is }
$$

$$
d \tau^{2}=\left(1-\frac{2 G M}{r c^{2}}\right) d t^{2}-\frac{1}{c^{2}} \frac{d r^{2}}{1-\frac{2 G M}{r c^{2}}}=d \hat{t}^{2}
$$

$$
-\frac{1}{c^{2}} d \hat{r}^{2}=d \hat{t}^{2}\left(1-\frac{V^{2}}{c^{2}}\right)
$$

Now,

$$
\begin{gathered}
\tau=\int d \tau=\int \frac{d \hat{t}}{\sqrt{1+\frac{a_{0}{ }^{2} \hat{t}^{2}}{c^{2}}}}=\frac{c}{a_{0}} \sinh ^{-1}\left(\frac{a_{0}}{c} \hat{t}\right), \\
\hat{t}=\frac{c}{a_{0}} \sinh \left(\frac{a_{0} \tau}{c}\right),
\end{gathered}
$$




$$
\begin{gathered}
\hat{r}=\int V d \hat{t}=\int \frac{a_{0} \hat{t} d \hat{t}}{\sqrt{1+\frac{a_{0}{ }^{2} \hat{t}^{2}}{c^{2}}}}=\frac{c^{2}}{a_{0}} \sqrt{1+\frac{a_{0}{ }^{2} \hat{t}^{2}}{c^{2}}} \\
=\frac{c^{2}}{a_{0}} \cosh \left(\frac{a_{0} \tau}{c}\right) \\
\frac{d \hat{t}}{d \tau}=\cosh \left(\frac{a_{0}}{c} \tau\right), \frac{1}{c} \frac{d \hat{r}}{d \tau}=\sinh \left(\frac{a_{0}}{c} \tau\right)
\end{gathered}
$$

\section{The Tetrad in Curved Space-time}

The tetrad $e_{\hat{\mu}}^{\hat{\alpha}}$ is the unit vector, which is defined by the following formula

$$
\eta_{\hat{\alpha} \hat{\beta}} e^{\hat{\alpha}} e_{\hat{\beta}}^{\hat{\beta}}=g_{\hat{\mu} \hat{v}}
$$

Now, if the matter moves along $\hat{r}$-axis in the curved space-time

$$
\eta_{\hat{\alpha} \hat{\beta}} e^{\hat{\alpha}}(\tau) e_{\hat{\mu}}^{\hat{\beta}}(\tau)=g_{\hat{\mu} \hat{v}}=\eta_{\hat{\mu} \hat{v}}, \quad g_{\hat{\mu} \hat{v}}=\eta_{\hat{\mu} \hat{v}}
$$

Hence, Eqn. (6), Eqn. (7) become

$$
\begin{gathered}
\eta_{\hat{\alpha} \hat{\beta}} e^{\hat{\alpha}_{\hat{o}}}(\tau) e^{\hat{\beta}_{\hat{0}}}(\tau)=\eta_{\hat{0} \hat{0}}=-1 \\
d \tau^{2}=-\frac{1}{c^{2}} \eta_{\hat{\alpha} \hat{\beta}} d \hat{x}^{\alpha} d \hat{x}^{\beta} \\
\rightarrow-1=\eta_{\hat{\alpha} \hat{\beta}}\left(\frac{1}{c} \frac{d \hat{x}^{\alpha}}{d \tau}\right)\left(\frac{1}{c} \frac{d \hat{x}^{\beta}}{d \tau}\right)=\eta_{\hat{\alpha} \hat{\beta}} e^{\hat{\alpha}} \hat{0}(\tau) e^{\hat{\beta}} \hat{0}(\tau) \\
\hat{x}^{\alpha}=(c \hat{t}, \hat{r}, \hat{\theta}, \hat{\phi})
\end{gathered}
$$

According to Eqn. (5) and Eqn. (9),

$$
e^{\hat{\alpha}_{\hat{o}}}(\tau)=\frac{1}{c} \frac{d \hat{x}^{\alpha}}{d \tau}=\left(\cosh \left(\frac{a_{0} \tau}{c}\right), \sinh \left(\frac{a_{0} \tau}{c}\right), 0,0\right)
$$

About $\hat{\theta}$-axis and $\hat{\phi}$-axis orientations, as

$$
\begin{aligned}
& \eta_{\hat{2} \hat{2}} e^{\hat{2}_{\hat{2}}}(\tau) e^{\hat{2}_{\hat{2}}}(\tau)=\eta_{\hat{2} \hat{2}}=1, \quad e^{\hat{\alpha}_{\hat{2}}}(\tau)=(0,0,1,0) \\
& \eta_{\hat{\hat{3}} \hat{\hat{3}}} e^{\hat{3}_{\hat{3}}}(\tau) e^{\hat{3}_{\hat{3}}}(\tau)=\eta_{\hat{\hat{3}} \hat{3}}=1, \quad e^{\hat{\alpha}_{\hat{3}}(\tau)=(0,0,0,1)}
\end{aligned}
$$

The other vector, $e^{\hat{\alpha}_{\hat{1}}}(\tau)$ has to satisfy the tetrad condition given by Eqn. (6) and Eqn. (7), and therefore we have

$$
e^{\hat{\alpha}_{\hat{1}}(\tau)}=\left(\sinh \left(\frac{a_{0} \tau}{c}\right), \cosh \left(\frac{a_{0} \tau}{c}\right), 0,0\right)
$$

Now, since

$$
\begin{aligned}
& \bar{e}_{\hat{t}}^{\rho}=\left(1 / \sqrt{1-\frac{2 G M}{r c^{2}}}, 0,0,0\right) \\
& \bar{e}_{\hat{r}}{ }^{\rho}=\left(0, \sqrt{1-\frac{2 G M}{r c^{2}}}, 0,0\right) \\
& \bar{e}_{\hat{\theta}}{ }^{\rho}=(0,0,1 / r, 0) \quad, \bar{e}_{\hat{\phi}}{ }^{\rho}=(0,0,0,1 / r \sin \theta) \\
& g_{\rho \sigma} \bar{e}_{\hat{\alpha}}{ }^{\rho} \bar{e}_{\hat{\beta}}{ }^{\sigma}=\eta_{\hat{\alpha} \hat{\beta}} \\
& \frac{a_{0}}{c} \hat{t}=\sinh \left(\frac{a_{0}}{c} \tau\right)=\frac{v / c}{\sqrt{1-v^{2} / c^{2}}}, \\
& \sqrt{1+\frac{a_{0}{ }^{2} \hat{t}^{2}}{c^{2}}}=\cosh \left(\frac{a_{0}}{c} \tau\right)=\frac{1}{\sqrt{1-v^{2} / c^{2}}}
\end{aligned}
$$

Therefore, the Lorentz transformation $B^{\hat{\alpha}} \hat{\mu}(v)$ is

$$
\begin{gathered}
B_{\hat{\mu}}^{\hat{\alpha}}(v)=\left(\begin{array}{cccc}
\frac{1}{\sqrt{1-v^{2} / c^{2}}} & \frac{v / c}{\sqrt{1-v^{2} / c^{2}}} & 0 & 0 \\
\frac{v / c}{\sqrt{1-v^{2} / c^{2}}} & \frac{1}{\sqrt{1-v^{2} / c^{2}}} & 0 & 0 \\
0 & 0 & 1 & 0 \\
0 & 0 & 0 & 1
\end{array}\right) \\
=e^{\hat{\alpha}} \hat{\mu}(\tau)=\left(\begin{array}{cccc}
\cosh \left(\frac{a_{0}}{c} \tau\right) & \sinh \left(\frac{a_{0}}{c} \tau\right) & 0 & 0 \\
\sinh \left(\frac{a_{0}}{c} \tau\right) & \cosh \left(\frac{a_{0}}{c} \tau\right) & 0 & 0 \\
0 & 0 & 1 & 0 \\
0 & 0 & 0 & 1
\end{array}\right) \\
\bar{e}_{\hat{\mu}}{ }^{\rho}=B_{\hat{\mu}}^{\hat{\alpha}}(v) \bar{e}_{\hat{\alpha}}{ }^{\rho}=e^{\hat{\alpha}}{ }_{\hat{\mu}}(\tau) \bar{e}_{\hat{\alpha}}{ }^{\rho}
\end{gathered}
$$

Hence,

$$
\begin{aligned}
& g_{\rho \sigma} \bar{e}_{\hat{\alpha}}{ }^{\rho} \bar{e}_{\hat{\beta}}{ }^{\sigma}=\eta_{\hat{\alpha} \hat{\beta}} \\
& g_{\rho \sigma} B^{\hat{\alpha}}{ }_{\hat{\mu}}(v) \bar{e}_{\hat{\alpha}}{ }^{\rho} B^{\hat{\beta}}{ }_{\hat{v}}(v) \bar{e}_{\hat{\beta}}{ }^{\sigma} \\
& \quad=g_{\rho \sigma} \bar{e}_{\hat{\mu}}{ }^{\rho} \bar{e}_{\hat{v}}{ }^{\sigma}=\eta_{\hat{\alpha} \hat{\beta}} e^{\hat{\alpha}}{ }_{\hat{\mu}}(\tau) e^{\hat{\beta}}{ }_{\hat{v}}(\tau)=\eta_{\hat{\mu} \hat{v}}
\end{aligned}
$$




\section{The Accelerated Frame in the Curved} Space-time

The accelerated frame $\hat{\xi}$ in the curved space-time can be defined as

$$
\begin{aligned}
d \tau^{2} & =-\frac{1}{c^{2}} \eta_{\hat{\alpha} \hat{\beta}} d \hat{x}^{\alpha} d \hat{x}^{\beta}=-\frac{1}{c^{2}} \eta_{\hat{\alpha} \hat{\beta}} \frac{\partial \hat{x}^{\alpha}}{\partial \hat{\xi}^{\mu}} \frac{\partial \hat{x}^{\beta}}{\partial \hat{\xi}^{v}} d \hat{\xi}^{\mu} d \hat{\xi}^{v} \\
& =-\frac{1}{c^{2}} \eta_{\hat{\alpha} \hat{\beta}} e^{\hat{\alpha}} e_{\hat{\mu}} e^{\hat{\beta}} d \hat{\xi}^{\mu} d \hat{\xi}^{v} \\
& =-\frac{1}{c^{2}} g_{\hat{\mu} \hat{v}} d \hat{\xi}^{\mu} d \hat{\xi}^{v} \\
e^{\hat{\alpha}} \hat{\mu} & =\frac{\partial \hat{x}^{\alpha}}{\partial \hat{\xi}^{\mu}}, \frac{\partial e^{\hat{\alpha}} \hat{\hat{0}}}{\partial \hat{\xi}^{1}}=\frac{\partial^{2} \hat{x}^{\alpha}}{c \partial \hat{\xi}^{0} \partial \hat{\xi}^{1}}=\frac{\partial e^{\hat{\alpha}_{\hat{1}}}}{c \partial \hat{\xi}^{0}}
\end{aligned}
$$

\subsection{Case-1}

Now, in Eqns. (10) - (12), if one uses $\hat{\xi}^{0}$ instead of $\tau$, then one can obtain new equations as

$$
\begin{aligned}
& e^{\hat{\alpha}_{\hat{0}}}\left(\hat{\xi}^{0}\right)=\frac{1}{c} \frac{\partial \hat{x}^{\alpha}}{\partial \hat{\xi}^{0}}=\left(\left(1+\frac{a_{0} \hat{\xi}^{1}}{c^{2}}\right) \cosh \left(\frac{a_{0} \hat{\xi}^{0}}{c}\right),\right. \\
& \left.\left(1+\frac{a_{0} \hat{\xi}^{1}}{c^{2}}\right) \sinh \left(\frac{a_{0} \hat{\xi}^{0}}{c}\right), 0,0\right) \\
& e^{\hat{\alpha}_{\hat{i}}}\left(\hat{\xi}^{0}\right)=\frac{\partial \hat{x}^{\alpha}}{\partial \hat{\xi}^{1}}=\left(\sinh \left(\frac{a_{0} \hat{\xi}^{0}}{c}\right), \cosh \left(\frac{a_{0} \hat{\xi}^{0}}{c}\right), 0,0\right) \\
& e^{\hat{\alpha}_{\hat{2}}}\left(\hat{\xi}^{0}\right)=\frac{\partial \hat{x}^{\alpha}}{\partial \hat{\xi}^{2}}=(0,0,1,0), \\
& e^{\hat{\alpha}_{\hat{\jmath}}}\left(\hat{\xi}^{0}\right)=\frac{\partial \hat{x}^{\alpha}}{\partial \hat{\xi}^{3}}=(0,0,1,0)
\end{aligned}
$$

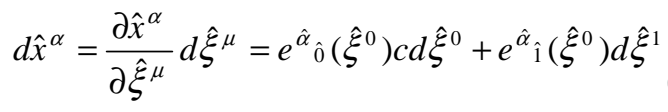

$$
\begin{aligned}
& +e^{\hat{\alpha}_{\hat{2}}}\left(\hat{\xi}^{0}\right) d \hat{\xi}^{2}+e^{\hat{\alpha}} \hat{\hat{\xi}}\left(\hat{\xi}^{0}\right) d \hat{\xi}^{3}
\end{aligned}
$$

Hence,

$$
\begin{aligned}
c d \hat{t}= & c d t \sqrt{1-\frac{2 G M}{r c^{2}}}=\left(1+\frac{a_{0} \hat{\xi}^{1}}{c^{2}}\right) \cosh \left(\frac{a_{0} \hat{\xi}^{0}}{c}\right) c d \hat{\xi}^{0} \\
& +\sinh \left(\frac{a_{0} \hat{\xi}^{0}}{c}\right) d \hat{\xi}^{1}
\end{aligned}
$$

$$
\begin{aligned}
& d \hat{r}=\frac{d r}{\sqrt{1-\frac{2 G M}{r c^{2}}}}=\left(1+\frac{a_{0} \hat{\xi}^{1}}{c^{2}}\right) \sinh \left(\frac{a_{0} \hat{\xi}^{0}}{c}\right) c d \hat{\xi}^{0} \\
& +\cosh \left(\frac{a_{0} \hat{\xi}^{0}}{c}\right) d \hat{\xi}^{1} \\
& d \hat{\boldsymbol{\theta}}=d \hat{\xi}^{2}, d \hat{\phi}=d \hat{\xi}^{3} \\
& d \tau^{2}=\left(1-\frac{2 G M}{r c^{2}}\right) d t^{2}-\frac{1}{c^{2}}\left[\frac{d r^{2}}{1-\frac{2 G M}{r c^{2}}}+r^{2} d \theta^{2}\right. \\
& \left.+r^{2} \sin ^{2} \theta d \phi^{2}\right] \\
& =d \hat{t}^{2}-\frac{1}{c^{2}}\left[d \hat{r}^{2}+d \hat{\theta}^{2}+d \hat{\phi}^{2}\right] \\
& =\left(1+\frac{a_{0} \hat{\xi}^{1}}{c^{2}}\right)^{2}\left(d \hat{\xi}^{0}\right)^{2}-\frac{1}{c^{2}}\left[\left(d \hat{\xi}^{1}\right)^{2}+\left(d \hat{\xi}^{2}\right)^{2}+\left(d \hat{\xi}^{3}\right)^{2}\right]
\end{aligned}
$$

The co-ordinate transformation is

$$
\begin{gathered}
c \hat{t}=\left(\frac{c^{2}}{a_{0}}+\hat{\xi}^{1}\right) \sinh \left(\frac{a_{0} \hat{\xi}^{0}}{c}\right), \\
\hat{r}=\left(\frac{c^{2}}{a_{0}}+\hat{\xi}^{1}\right) \cosh \left(\frac{a_{0} \hat{\xi}^{0}}{c}\right)-\frac{c^{2}}{a_{0}} \\
\hat{\theta}=\hat{\xi}^{2}, \hat{\phi}=\hat{\xi}^{3}
\end{gathered}
$$

The inverse-transformation is

$$
\begin{gathered}
\hat{\xi}^{0}=\frac{c}{a_{0}} \tanh ^{-1}\left(\frac{c \hat{t}}{\hat{r}+\frac{c^{2}}{a_{0}}}\right), \\
\hat{\xi}^{1}=\sqrt{\left(\hat{r}+\frac{c^{2}}{a_{0}}\right)^{2}-c^{2} \hat{t}^{2}}-\frac{c^{2}}{a_{0}} \\
\hat{\xi}^{2}=\hat{\theta}, \hat{\xi}^{3}=\hat{\phi}
\end{gathered}
$$

We now calculate the curvature tensor $R_{\hat{\mu} \hat{p} \hat{p}}(\hat{\xi})$ thus obtaining the following expression 


$$
\begin{aligned}
& R_{\hat{\mu} \hat{v} \hat{\rho} \hat{\lambda}}(\hat{\xi})=\frac{\partial \hat{x}^{\alpha}}{\partial \hat{\xi}^{\mu}} \frac{\partial \hat{x}^{\beta}}{\partial \hat{\xi}^{v}} \frac{\partial \hat{x}^{\gamma}}{\partial \hat{\xi}^{\rho}} \frac{\partial \hat{x}^{\delta}}{\partial \hat{\xi}^{\lambda}} R_{\hat{\alpha} \hat{\beta} \hat{\gamma} \hat{\delta}}(\hat{X}) \\
& =e^{\hat{\alpha}_{\mu}}\left(\hat{\xi}^{0}\right) e^{\hat{\beta}} \hat{v}\left(\hat{\xi}^{0}\right) e^{\hat{\gamma}} \hat{\rho}\left(\hat{\xi}^{0}\right) e^{\hat{\delta}} \hat{\lambda}\left(\hat{\xi}^{0}\right) R_{\hat{\alpha} \hat{\beta} \hat{\gamma} \hat{\delta}}(\hat{X}) \\
& R_{\hat{t} \hat{r} \hat{r} \hat{r}}=-R_{\hat{t} \hat{r} \hat{r} \hat{t}}=R_{\hat{r} \hat{r} \hat{t} \hat{t}}=-R_{\hat{r} \hat{t} \hat{r} \hat{r}}=\frac{2 G M}{r^{3} c^{2}}, \\
& R_{\hat{t} \hat{\theta} \hat{\theta} \hat{\theta}}=-R_{\hat{t} \hat{\theta} \hat{\theta} \hat{t}}=R_{\hat{\theta} \hat{t} \hat{\theta} \hat{t}}=-R_{\hat{\theta} \hat{t} \hat{t} \hat{\theta}}=-\frac{G M}{r^{3} c^{2}}=R_{\hat{t} \hat{\phi} \hat{x} \hat{\phi}} \\
& =-R_{\hat{t} \hat{\phi} \hat{\phi} \hat{t}}=R_{\hat{\hat{t} \hat{\phi} \hat{\phi} t}}=-R_{\hat{\phi} \hat{t} \hat{\phi} \hat{\phi}} \\
& R_{\hat{\theta} \hat{\phi} \hat{\theta} \hat{\phi}}=-R_{\hat{\theta} \hat{\phi} \hat{\phi} \hat{\theta}}=R_{\hat{\theta} \hat{\phi} \hat{\theta} \hat{\phi}}=-R_{\hat{\theta} \hat{\phi} \hat{\phi} \hat{\theta}}=-\frac{2 G M}{r^{3} c^{2}} \\
& R_{\hat{r} \hat{r} \hat{r} \hat{\theta}}=-R_{\hat{r} \hat{\theta} \hat{\theta} \hat{r}}=R_{\hat{\theta} \hat{r} \hat{\theta} \hat{r}}=-R_{\hat{\theta} \hat{r} \hat{r} \hat{\theta}}=\frac{G M}{r^{3} c^{2}}=R_{\hat{r} \hat{\phi} \hat{r} \hat{\theta}} \\
& =-R_{\hat{r} \hat{\phi} \hat{\phi} \hat{r}}=R_{\hat{\phi} \hat{r} \hat{\phi} \hat{r}}=-R_{\hat{\phi} \hat{r} \hat{r} \hat{\phi}}
\end{aligned}
$$

Therefore,

$$
\begin{aligned}
& e^{\hat{\alpha}_{0}}\left(\hat{\xi}^{0}\right)=\left(\left(1+\frac{a_{0} \hat{\xi}^{1}}{c^{2}}\right) \cosh \left(\frac{a_{0} \hat{\xi}^{0}}{c}\right),\right. \\
& \left.\left(1+\frac{a_{0} \hat{\xi}^{1}}{c^{2}}\right) \sinh \left(\frac{a_{0} \hat{\xi}^{0}}{c}\right), 0,0\right) \\
& e^{\hat{\alpha}_{\hat{1}}}\left(\hat{\xi}^{0}\right)=\left(\sinh \left(\frac{a_{0} \hat{\xi}^{0}}{c}\right), \cosh \left(\frac{a_{0} \hat{\xi}^{0}}{c}\right), 0,0\right)
\end{aligned}
$$

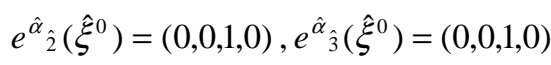

$$
\begin{aligned}
& R_{\hat{0} \hat{1} \hat{0} \hat{1}}(\hat{\xi})=\frac{2 G M}{r^{3} c^{2}}\left(1+\frac{a_{0} \hat{\xi}^{1}}{c^{2}}\right)^{2}, \\
& R_{\hat{0} \hat{2} \hat{0} \hat{2}}(\hat{\xi})=R_{\hat{0} \hat{3} \hat{0} \hat{3}}(\hat{\xi})=-\frac{G M}{r^{3} c^{2}}\left(1+\frac{a_{0} \hat{\xi}^{1}}{c^{2}}\right)^{2} \\
& R_{\hat{\hat{z}} \hat{2} \hat{2} \hat{3}}(\hat{\xi})=-\frac{2 G M}{r^{3} c^{2}}, \\
& R_{\hat{1} \hat{2} \hat{1} \hat{2}}(\hat{\xi})=R_{\hat{1} \hat{1} \hat{1} \hat{3}}(\hat{\xi})=\frac{G M}{r^{3} c^{2}}
\end{aligned}
$$

$$
a=\frac{d}{d t}\left(\frac{u}{\sqrt{1-\frac{2 G M}{r c^{2}}-\frac{u^{2}}{c^{2}}}}\right)
$$

This is equal to $-g$, the pure gravity acceleration. In Eqn. (33), $r_{0}$ is the location of the stationary accelerated frame.

In the curved space-time, the curvature tensor $R_{\hat{\mu} \hat{\nu} \hat{\imath} \hat{\imath}}(\hat{\xi})$ of the stationary accelerated frame is

Specially, when $t=0$,

$$
\begin{aligned}
R_{\hat{0} \hat{1} \hat{1}}(\hat{\xi})= & \frac{2 G M}{r^{3} c^{2}}\left(1+\frac{a_{0} \hat{\xi}^{1}}{c^{2}}\right)^{2}=\frac{2 G M}{r^{3} c^{2}}\left(1+\frac{a_{0} \hat{r}}{c^{2}}\right)^{2} \\
& =\frac{2 G M}{r^{3} c^{2}}\left[1+\frac{a_{0}}{c^{2}}\left\{\sqrt{r} \sqrt{r-\frac{2 G M}{c^{2}}}+\frac{2 G M}{c^{2}} \ln \left|\sqrt{r}+\sqrt{r-\frac{2 G M}{c^{2}}}\right|\right.\right. \\
& \left.\left.-\sqrt{r_{0}} \sqrt{r_{0}-\frac{2 G M}{c^{2}}}-\frac{2 G M}{c^{2}} \ln \left|\sqrt{r_{0}}+\sqrt{r_{0}-\frac{2 G M}{c^{2}}}\right|\right\}\right]^{2}
\end{aligned}
$$




$$
\begin{aligned}
& =\frac{2 G M}{r^{3} c^{2}}\left[1+\frac{1}{c^{2}} \frac{a}{\sqrt{1-\frac{2 G M}{r c^{2}}}}\left\{\sqrt{r} \sqrt{r-\frac{2 G M}{c^{2}}}+\frac{2 G M}{c^{2}} \ln \left|\sqrt{r}+\sqrt{r-\frac{2 G M}{c^{2}}}\right|\right.\right. \\
& \left.\left.-\sqrt{r_{0}} \sqrt{r_{0}-\frac{2 G M}{c^{2}}}-\frac{2 G M}{c^{2}} \ln \left|\sqrt{r_{0}}+\sqrt{r_{0}-\frac{2 G M}{c^{2}}}\right|\right\}\right]^{2} \\
& R_{\hat{0} \hat{0} \hat{0} \hat{2}}(\hat{\xi})=R_{\hat{0} \hat{3} \hat{0} \hat{3}}(\hat{\xi})=-\frac{G M}{r^{3} c^{2}}\left(1+\frac{a_{0} \hat{\xi}^{1}}{c^{2}}\right)^{2}=-\frac{G M}{r^{3} c^{2}}\left(1+\frac{a_{0} \hat{r}}{c^{2}}\right)^{2} \\
& =-\frac{G M}{r^{3} c^{2}}\left[1+\frac{a_{0}}{c^{2}}\left\{\sqrt{r} \sqrt{r-\frac{2 G M}{c^{2}}}+\frac{2 G M}{c^{2}} \ln \left|\sqrt{r}+\sqrt{r-\frac{2 G M}{c^{2}}}\right|\right.\right. \\
& \left.\left.-\sqrt{r_{0}} \sqrt{r_{0}-\frac{2 G M}{c^{2}}}-\frac{2 G M}{c^{2}} \ln \left|\sqrt{r_{0}}+\sqrt{r_{0}-\frac{2 G M}{c^{2}}}\right|\right\}\right]^{2} \\
& =-\frac{G M}{r^{3} c^{2}}\left[1+\frac{1}{c^{2}} \frac{a}{\sqrt{1-\frac{2 G M}{r c^{2}}}}\left\{\sqrt{r} \sqrt{r-\frac{2 G M}{c^{2}}}+\frac{2 G M}{c^{2}} \ln \left|\sqrt{r}+\sqrt{r-\frac{2 G M}{c^{2}}}\right|\right.\right. \\
& \left.\left.-\sqrt{r_{0}} \sqrt{r_{0}-\frac{2 G M}{c^{2}}}-\frac{2 G M}{c^{2}} \ln \left|\sqrt{r_{0}}+\sqrt{r_{0}-\frac{2 G M}{c^{2}}}\right|\right\}\right]^{2} \\
& R_{\hat{2} \hat{3} \hat{2} \hat{3}}(\hat{\xi})=-\frac{2 G M}{r^{3} c^{2}}, \quad R_{\hat{1} \hat{2} \hat{1} \hat{2}}(\hat{\xi})=R_{\hat{1} \hat{3} \hat{1} \hat{3}}(\hat{\xi})=\frac{G M}{r^{3} c^{2}}
\end{aligned}
$$

\subsection{Case-2}

Now, in Eqns. (10), (11) and (12), if one uses $\hat{\xi}^{0}$ instead of $\tau$ and multiply by $\exp \left(\frac{a_{0}}{c^{2}} \hat{\xi}^{1}\right)$

$$
\begin{aligned}
& e^{\hat{\alpha}_{\hat{o}}}\left(\hat{\xi}^{0}\right)=\frac{1}{c} \frac{\partial \hat{x}^{\alpha}}{\partial \hat{\xi}^{0}}=\left(\exp \left(\frac{a_{0}}{c^{2}} \hat{\xi}^{1}\right) \cosh \left(\frac{a_{0} \hat{\xi}^{0}}{c}\right),\right. \\
& \left.\exp \left(\frac{a_{0}}{c^{2}} \hat{\xi}^{1}\right) \sinh \left(\frac{a_{0} \hat{\xi}^{0}}{c}\right), 0,0\right) \\
& e^{\hat{\alpha}_{\hat{1}}}\left(\hat{\xi}^{0}\right)=\frac{\partial \hat{x}^{\alpha}}{\partial \hat{\xi}^{1}}=\left(\exp \left(\frac{a_{0}}{c^{2}} \hat{\xi}^{1}\right) \sinh \left(\frac{a_{0} \hat{\xi}^{0}}{c}\right),\right. \\
& \left.\exp \left(\frac{a_{0}}{c^{2}} \hat{\xi}^{1}\right) \cosh \left(\frac{a_{0} \hat{\xi}^{0}}{c}\right), 0,0\right) \\
& e^{\hat{\alpha}} \hat{2}\left(\hat{\xi}^{0}\right)=\frac{\partial \hat{x}^{\alpha}}{\partial \hat{\xi}^{2}}=(0,0,1,0), \\
& e^{\hat{\alpha}_{\hat{3}}}\left(\hat{\xi}^{0}\right)=\frac{\partial \hat{x}^{\alpha}}{\partial \hat{\xi}^{3}}=(0,0,1,0)
\end{aligned}
$$

$$
\begin{aligned}
& d \hat{x}^{\alpha}=\frac{\partial \hat{x}^{\alpha}}{\partial \hat{\xi}^{\mu}} d \hat{\xi}^{\mu}=e^{\hat{\alpha}_{\hat{0}}}\left(\hat{\xi}^{0}\right) c d \hat{\xi}^{0}+e^{\hat{\alpha}_{\hat{1}}}\left(\hat{\xi}^{0}\right) d \hat{\xi}^{1} \\
& +e^{\hat{\alpha}_{\hat{2}}}\left(\hat{\xi}^{0}\right) d \hat{\xi}^{2}+e^{\hat{\alpha}_{\hat{z}}}\left(\hat{\xi}^{0}\right) d \hat{\xi}^{3}
\end{aligned}
$$

Hence,

$$
\begin{gathered}
c d \hat{t}=c d t \sqrt{1-\frac{2 G M}{r c^{2}}}=\exp \left(\frac{a_{0}}{c^{2}} \hat{\xi}^{1}\right) \cosh \left(\frac{a_{0} \hat{\xi}^{0}}{c}\right) c d \hat{\xi}^{0} \\
+\exp \left(\frac{a_{0}}{c^{2}} \hat{\xi}^{1}\right) \sinh \left(\frac{a_{0} \hat{\xi}^{0}}{c}\right) d \hat{\xi}^{1} \\
d \hat{r}=\frac{d r}{\sqrt{1-\frac{2 G M}{r c^{2}}}}=\exp \left(\frac{a_{0}}{c^{2}} \hat{\xi}^{1}\right) \sinh \left(\frac{a_{0} \hat{\xi}^{0}}{c}\right) c d \hat{\xi}^{0} \\
+\exp \left(\frac{a_{0}}{c^{2}} \hat{\xi}^{1}\right) \cosh \left(\frac{a_{0} \hat{\xi}^{0}}{c}\right) d \hat{\xi}^{1} \\
d \hat{\theta}=d \hat{\xi}^{2} \quad, \quad d \hat{\phi}=d \hat{\xi}^{3}
\end{gathered}
$$




$$
\begin{aligned}
d \tau^{2}= & \left(1-\frac{2 G M}{r c^{2}}\right) d t^{2} \\
& -\frac{1}{c^{2}}\left[\frac{d r^{2}}{1-\frac{2 G M}{r c^{2}}}+r^{2} d \theta^{2}+r^{2} \sin ^{2} \theta d \phi^{2}\right] \\
& =d \hat{t}^{2}-\frac{1}{c^{2}}\left[d \hat{r}^{2}+d \hat{\theta}^{2}+d \hat{\phi}^{2}\right] \\
= & \exp \left(2 \frac{a_{0} \hat{\xi}^{1}}{c^{2}}\right)\left(d \hat{\xi}^{0}\right)^{2} \\
& -\frac{1}{c^{2}}\left[\exp \left(2 \frac{a_{0} \hat{\xi}^{1}}{c^{2}}\right)\left(d \hat{\xi}^{1}\right)^{2}+\left(d \hat{\xi}^{2}\right)^{2}+\left(d \hat{\xi}^{3}\right)^{2}\right]
\end{aligned}
$$

The coordinate transformation is

$$
\begin{aligned}
& c \hat{t}=\frac{c^{2}}{a_{0}} \exp \left(\frac{a_{0}}{c^{2}} \hat{\xi}^{1}\right) \sinh \left(\frac{a_{0} \hat{\xi}^{0}}{c}\right), \\
& \hat{r}=\frac{c^{2}}{a_{0}} \exp \left(\frac{a_{0}}{c^{2}} \hat{\xi}^{1}\right) \cosh \left(\frac{a_{0} \hat{\xi}^{0}}{c}\right)-\frac{c^{2}}{a_{0}} \\
& \hat{\theta}=\hat{\xi}^{2}, \hat{\phi}=\hat{\xi}^{3}
\end{aligned}
$$

The inverse-transformation is

$$
\begin{aligned}
& \hat{\xi}^{0}=\frac{c}{a_{0}} \tanh ^{-1}\left(\frac{c \hat{t}}{\hat{r}+\frac{c^{2}}{a_{0}}}\right), \\
& \hat{\xi}^{1}=\frac{c^{2}}{a_{0}} \ln \left|\frac{a_{0}}{c^{2}} \sqrt{\left(\hat{r}+\frac{c^{2}}{a_{0}}\right)^{2}-c^{2} \hat{t}^{2}}\right| \\
& \hat{\xi}^{2}=\hat{\theta}, \hat{\xi}^{3}=\hat{\phi}
\end{aligned}
$$

If we calculate the curvature tensor $R_{\hat{\mu} \hat{v} \hat{\rho} \hat{\lambda}}(\hat{\xi})$ as

$$
\begin{aligned}
R_{\hat{\mu} \hat{v} \hat{\rho} \hat{\lambda}}(\hat{\xi}) & =\frac{\partial \hat{x}^{\alpha}}{\partial \hat{\xi}^{\mu}} \frac{\partial \hat{x}^{\beta}}{\partial \hat{\xi}^{v}} \frac{\partial \hat{x}^{\gamma}}{\partial \hat{\xi}^{\rho}} \frac{\partial \hat{x}^{\delta}}{\partial \hat{\xi}^{\lambda}} R_{\hat{\alpha} \hat{\beta} \hat{\gamma} \delta}(\hat{X}) \\
& =e^{\hat{\alpha}}{ }_{\hat{\mu}}\left(\hat{\xi}^{0}\right) e^{\hat{\beta}} \hat{v}\left(\hat{\xi}^{0}\right) e^{\hat{\gamma}}\left(\hat{\xi}^{0}\right) e^{\hat{\delta}} \hat{\lambda}\left(\hat{\xi}^{0}\right) R_{\hat{\alpha} \hat{\beta} \hat{\gamma} \delta}(\hat{X})
\end{aligned}
$$

$$
R_{\hat{t} \hat{r} \hat{r} \hat{r}}=-R_{\hat{t} \hat{r} \hat{r} \hat{t}}=R_{\hat{\hat{t} \hat{t} \hat{r} \hat{t}}}=-R_{\hat{\hat{t} \hat{t} \hat{r} \hat{r}}}=\frac{2 G M}{r^{3} c^{2}},
$$

$$
\begin{aligned}
& R_{\hat{t} \hat{\theta} \hat{t} \hat{\theta}}=-R_{\hat{t} \hat{\theta} \hat{\theta} \hat{t}}=R_{\hat{\theta} \hat{t} \hat{\theta} \hat{t}}=-R_{\hat{\theta} \hat{t} \hat{t} \hat{\theta}}=-\frac{G M}{r^{3} c^{2}}=R_{\hat{t} \hat{\phi} \hat{t} \hat{\phi}} \\
& =-R_{\hat{t} \hat{\phi} \hat{\phi} \hat{t}}=R_{\hat{\phi} \hat{t} \hat{\phi} \hat{t}}=-R_{\hat{\phi} \hat{t} \hat{t} \hat{\phi}} \\
& R_{\hat{\theta} \hat{\phi} \hat{\theta} \hat{\phi}}=-R_{\hat{\theta} \hat{\phi} \hat{\phi} \hat{\theta}}=R_{\hat{\theta} \hat{\phi} \hat{\theta} \hat{\phi}}=-R_{\hat{\theta} \hat{\phi} \hat{\phi} \hat{\theta}}=-\frac{2 G M}{r^{3} c^{2}} \\
& R_{\hat{r} \hat{\theta} \hat{r} \hat{\theta}}=-R_{\hat{r} \hat{\theta} \hat{\theta} \hat{r}}=R_{\hat{\theta} \hat{r} \hat{\theta} \hat{r}}=-R_{\hat{\theta} \hat{r} \hat{r} \hat{\theta}}=\frac{G M}{r^{3} c^{2}}=R_{\hat{r} \hat{\phi} \hat{r} \hat{\phi}} \\
& =-R_{\hat{r} \hat{\phi} \hat{\phi} \hat{r}}=R_{\hat{\phi} \hat{r} \hat{\phi} \hat{r}}=-R_{\hat{\phi} \hat{r} \hat{r} \hat{\phi}}
\end{aligned}
$$

Then,

$$
\begin{aligned}
& e^{\hat{\alpha}_{\hat{0}}}\left(\hat{\xi}^{0}\right)=\left(\exp \left(\frac{a_{0}}{c^{2}} \hat{\xi}^{1}\right) \cosh \left(\frac{a_{0} \hat{\xi}^{0}}{c}\right),\right. \\
& \left.\exp \left(\frac{a_{0}}{c^{2}} \hat{\xi}^{1}\right) \sinh \left(\frac{a_{0} \hat{\xi}^{0}}{c}\right), 0,0\right) \\
& e^{\hat{\alpha}_{\hat{1}}}\left(\hat{\xi}^{0}\right)=\left(\exp \left(\frac{a_{0}}{c^{2}} \hat{\xi}^{1}\right) \sinh \left(\frac{a_{0} \hat{\xi}^{0}}{c}\right),\right. \\
& \left.\exp \left(\frac{a_{0}}{c^{2}} \hat{\xi}^{1}\right) \cosh \left(\frac{a_{0} \hat{\xi}^{0}}{c}\right), 0,0\right) \\
& e^{\hat{\alpha}_{\hat{2}}}\left(\hat{\xi}^{0}\right)=(0,0,1,0), e^{\hat{\alpha}_{\hat{3}}}\left(\hat{\xi}^{0}\right)=(0,0,1,0)
\end{aligned}
$$

$$
\begin{aligned}
& R_{\hat{0} \hat{1} \hat{0} \hat{1}}(\hat{\xi})=\frac{2 G M}{r^{3} c^{2}} \exp \left(4 \frac{a_{0} \hat{\xi}^{1}}{c^{2}}\right), \\
& R_{\hat{0} \hat{2} \hat{0} \hat{2}}(\hat{\xi})=R_{\hat{0} \hat{3} \hat{0} \hat{3}}(\hat{\xi})=-\frac{G M}{r^{3} c^{2}} \exp \left(2 \frac{a_{0} \hat{\xi}^{1}}{c^{2}}\right) \\
& R_{\hat{2} \hat{2} \hat{2} \hat{3}}(\hat{\xi})=-\frac{2 G M}{r^{3} c^{2}}, \\
& R_{\hat{1} \hat{2} \hat{1} \hat{2}}(\hat{\xi})=R_{\hat{1} \hat{z} \hat{1} \hat{3}}(\hat{\xi})=\frac{G M}{r^{3} c^{2}} \exp \left(2 \frac{a_{0}}{c^{2}} \hat{\xi}^{1}\right)
\end{aligned}
$$

As a special case, when $t=0$, we obtain

$$
\begin{aligned}
& u=\frac{1}{\sqrt{1-\frac{2 G M}{r c^{2}}} \frac{d r}{d t}=0 \rightarrow} \\
& V=\frac{d \hat{r}}{d \hat{t}}=\frac{a_{0} \hat{t}}{\sqrt{1+\frac{a_{0}{ }^{2} \hat{t}^{2}}{c^{2}}}}=\frac{d r}{d t} \frac{1}{\left(1-\frac{2 G M}{r c^{2}}\right)}=0
\end{aligned}
$$

Therefore, if, $t=\hat{t}=\hat{\xi}^{0}=0$, the theory treats the real situation. 


$$
\begin{aligned}
& \hat{\xi}^{1}=\frac{c^{2}}{a_{0}} \ln \left|\frac{a_{0}}{c^{2}} \sqrt{\left(\hat{r}+\frac{c^{2}}{a_{0}}\right)^{2}-c^{2} \hat{t}^{2}}\right|=\frac{c^{2}}{a_{0}} \ln \left|\left(1+\frac{a_{0}}{c^{2}} \hat{r}\right)\right| \\
& \exp \left(\frac{a_{0}}{c^{2}} \hat{\xi}^{1}\right)=1+\frac{a_{0}}{c^{2}} \hat{r} \\
& d \hat{r}=\frac{d r}{\sqrt{1-\frac{2 G M}{r c^{2}}}} \rightarrow \hat{r}=\sqrt{r} \sqrt{r-\frac{2 G M}{c^{2}}}+\frac{2 G M}{c^{2}} \ln \left|\sqrt{r}+\sqrt{r-\frac{2 G M}{c^{2}}}\right| \\
& -\sqrt{r_{0}} \sqrt{r_{0}-\frac{2 G M}{c^{2}}}-\frac{2 G M}{c^{2}} \ln \left|\sqrt{r_{0}}+\sqrt{r_{0}-\frac{2 G M}{c^{2}}}\right| \\
& a_{0}=\frac{1}{\sqrt{1-\frac{2 G M}{r c^{2}}}} \frac{d}{d t}\left(\frac{u}{\sqrt{1-\frac{2 G M}{r c^{2}}-\frac{u^{2}}{c^{2}}}}\right)=\frac{a}{\sqrt{1-\frac{2 G M}{r c^{2}}}} \\
& a=\frac{d}{d t}\left(\frac{u}{\sqrt{1-\frac{2 G M}{r c^{2}}-\frac{u^{2}}{c^{2}}}}\right)
\end{aligned}
$$

Now, in the curved space-time, the curvature tensor $R_{\hat{\mu} \hat{\rho} \hat{\rho} \hat{\lambda}}(\hat{\xi})$ of the stationary accelerated frame is

$$
\begin{aligned}
& R_{\hat{0} \hat{1} \hat{1} \hat{1}}(\hat{\xi})=\frac{2 G M}{r^{3} c^{2}} \exp \left(4 \frac{a_{0} \hat{\xi}^{1}}{c^{2}}\right)=\frac{2 G M}{r^{3} c^{2}}\left(1+\frac{a_{0}}{c^{2}} \hat{r}\right)^{4} \\
& =\frac{2 G M}{r^{3} c^{2}}\left[1+\frac{a_{0}}{c^{2}}\left\{\sqrt{r} \sqrt{r-\frac{2 G M}{c^{2}}}+\frac{2 G M}{c^{2}} \ln \left|\sqrt{r}+\sqrt{r-\frac{2 G M}{c^{2}}}\right|\right.\right. \\
& \left.\left.-\sqrt{r_{0}} \sqrt{r_{0}-\frac{2 G M}{c^{2}}}-\frac{2 G M}{c^{2}} \ln \left|\sqrt{r_{0}}+\sqrt{r_{0}-\frac{2 G M}{c^{2}}}\right|\right\}\right]^{4} \\
& =\frac{2 G M}{r^{3} c^{2}}\left[1+\frac{1}{c^{2}} \frac{a}{\sqrt{1-\frac{2 G M}{r c^{2}}}}\left\{\sqrt{r} \sqrt{r-\frac{2 G M}{c^{2}}}+\frac{2 G M}{c^{2}} \ln \left|\sqrt{r}+\sqrt{r-\frac{2 G M}{c^{2}}}\right|\right.\right. \\
& \left.\left.-\sqrt{r_{0}} \sqrt{r_{0}-\frac{2 G M}{c^{2}}}-\frac{2 G M}{c^{2}} \ln \left|\sqrt{r_{0}}+\sqrt{r_{0}-\frac{2 G M}{c^{2}}}\right|\right\}\right]^{4} \\
& R_{\hat{0} \hat{2} \hat{0} \hat{2}}(\hat{\xi})=R_{\hat{0} \hat{0} \hat{0} \hat{3}}(\hat{\xi})=-\frac{G M}{r^{3} c^{2}} \exp \left(2 \frac{a_{0} \hat{\xi}^{1}}{c^{2}}\right)=-\frac{G M}{r^{3} c^{2}}\left(1+\frac{a_{0} \hat{r}}{c^{2}}\right)^{2} \\
& =-\frac{G M}{r^{3} c^{2}}\left[1+\frac{a_{0}}{c^{2}}\left\{\sqrt{r} \sqrt{r-\frac{2 G M}{c^{2}}}+\frac{2 G M}{c^{2}} \ln \left|\sqrt{r}+\sqrt{r-\frac{2 G M}{c^{2}}}\right|\right.\right. \\
& \left.\left.-\sqrt{r_{0}} \sqrt{r_{0}-\frac{2 G M}{c^{2}}}-\frac{2 G M}{c^{2}} \ln \left|\sqrt{r_{0}}+\sqrt{r_{0}-\frac{2 G M}{c^{2}}}\right|\right\}\right]^{2} \\
& =-\frac{G M}{r^{3} c^{2}}\left[1+\frac{1}{c^{2}} \frac{a}{\sqrt{1-\frac{2 G M}{r c^{2}}}}\left\{\sqrt{r} \sqrt{r-\frac{2 G M}{c^{2}}}+\frac{2 G M}{c^{2}} \ln \left|\sqrt{r}+\sqrt{r-\frac{2 G M}{c^{2}}}\right|\right.\right. \\
& \left.\left.-\sqrt{r_{0}} \sqrt{r_{0}-\frac{2 G M}{c^{2}}}-\frac{2 G M}{c^{2}} \ln \left|\sqrt{r_{0}}+\sqrt{r_{0}-\frac{2 G M}{c^{2}}}\right|\right\}\right]^{2}
\end{aligned}
$$




$$
\begin{aligned}
& R_{\hat{2} \hat{3} \hat{2} \hat{3}}(\hat{\xi})=-\frac{2 G M}{r^{3} c^{2}}, \\
& R_{\hat{1} \hat{2} \hat{1} \hat{2}}(\hat{\xi})=R_{\hat{1} \hat{3} \hat{1} \hat{3}}(\hat{\xi})=\frac{G M}{r^{3} c^{2}} \exp \left(2 \frac{a_{0}}{c^{2}} \hat{\xi}^{1}\right)=\frac{G M}{r^{3} c^{2}}\left(1+\frac{a_{0}}{c^{2}} \hat{r}\right)^{2} \\
& =\frac{G M}{r^{3} c^{2}}\left[1+\frac{a_{0}}{c^{2}}\left\{\sqrt{r} \sqrt{r-\frac{2 G M}{c^{2}}}+\frac{2 G M}{c^{2}} \ln \left|\sqrt{r}+\sqrt{r-\frac{2 G M}{c^{2}}}\right|\right.\right. \\
& \left.\left.-\sqrt{r_{0}} \sqrt{r_{0}-\frac{2 G M}{c^{2}}}-\frac{2 G M}{c^{2}} \ln \left|\sqrt{r_{0}}+\sqrt{r_{0}-\frac{2 G M}{c^{2}}}\right|\right\}\right]^{2} \\
& =\frac{G M}{r^{3} c^{2}}\left[1+\frac{1}{c^{2}} \frac{a}{\sqrt{1-\frac{2 G M}{r c^{2}}}}\left\{\sqrt{r} \sqrt{r-\frac{2 G M}{c^{2}}}+\frac{2 G M}{c^{2}} \ln \left|\sqrt{r}+\sqrt{r-\frac{2 G M}{c^{2}}}\right|\right.\right. \\
& \left.\left.-\sqrt{r_{0}} \sqrt{r_{0}-\frac{2 G M}{c^{2}}}-\frac{2 G M}{c^{2}} \ln \left|\sqrt{r_{0}}+\sqrt{r_{0}-\frac{2 G M}{c^{2}}}\right|\right\}\right]^{2}
\end{aligned}
$$

\section{Conclusion}

In the general relativity theory, we define the accelerated frame that moves in $\hat{r}$-axis in the curved time-space. Specially, if, $t=\hat{t}=\hat{\xi}^{0}=0$, this theory treats the curvature tensor of the stationary accelerated frame in the curved spacetime in two-cases. In this context, the curvature tensor is divided into two parts: $R_{\hat{\mu} \hat{v} \hat{\rho} \hat{\lambda}}(\hat{\xi})$ is the curvature tensor as observed by the observer and $R_{\hat{\alpha} \hat{\beta} \hat{\gamma} \delta}(\hat{X})$ is the curvature tensor of the observer's own planet in the gravity field.

\section{References}

[1] S. Weinberg, Gravitation and Cosmology (John Wiley \& Sons Inc., 1972).

[2] P. Bergman, Introduction to the Theory of Relativity (Dover Pub. Co. Inc., New York, 1976) Chapter V.

[3] C. Misner, K. Thorne and J. Wheeler, Gravitation (W. H. Freedman \& Co., 1973).

[4] S. Hawking and G. Ellis, The Large Scale Structure of Space-Time (Cambridge University Press, 1973).

[5] R. Adler, M. Bazin and M. Schiffer, Introduction to General Relativity (McGraw-Hill Inc., 1965).

[6] M. Schwarzschild, Structure and Evolution of the Stars (Princeton University Press, 1958), reprint (Dover, N.Y., 1965) Chapter II.

[7] S. Chandrasekhar, Mon. Not. Roy. Astron. Soc. 95, 207 (1935).

[8] C. Rhoades, "Investigations in the Physics of Neutron Stars", doctoral dissertation, Princeton University.

[9] J. Oppenheimer and H. Snyder, Phys. Rev. 56, 455 (1939). 